\title{
Alternative prey influence the predation of mosquito larvae by three water bug species (Heteroptera: Nepidae)
}

\author{
Nabaneeta Saha', Malini Kundu², Goutam K. Saha', Gautam Aditya ${ }^{1,2, *}$ \\ ${ }^{1}$ Department of Zoology, University of Calcutta, 35 Ballygunge Circular Road, Kolkata 700019, India, \\ e-mail: nsaha790@gmail.com (N.S.); gkszoo@rediffmail.com (G.K.S.); \\ gautamaditya2001@gmail.com (*corresponding author, G.A.) \\ ${ }^{2}$ Department of Zoology, University of Burdwan, Golapbag, Burdwan 713104, India, e-mail: malinikundu@rediffmail.com
}

\begin{abstract}
The indirect interactions among multiple prey sharing a common predator characterize apparent competition. In conservation biological control involving mosquitoes and controphic prey against generalist insect predators, apparent competition may be a crucial factor determining the extent of success. The possible influence of apparent competition on mosquito prey consumption by three water bugs (Heteroptera: Nepidae): Ranatra elongata, Ranatra filiformis, and Laccotrephes griseus was assessed under laboratory conditions. Tadpoles (Duttaphrynus melanostictus), chironomid larvae, snails (Racesina luteola) and fish fingerlings (Labeo rohita) were considered as alternative prey under two prey or multi prey conditions against instar IV larvae of Culex quinquefasciatus as target prey. Under two prey conditions all the predators exhibited a preference (significant Manly's $\alpha$ ) for mosquito larvae, against fish fingerlings by R. filiformis. In the presence of multiple prey, mosquito larvae were selected by the predators. Using the ratio of mosquito selectivity in two prey and multi-prey conditions as a measure of apparent competition, chironomid larvae had greatest effect in suppressing mosquito selectivity for $R$. elongata, and fish fingerlings for $R$. filiformis and $L$. griseus. It seems that the prey preference of $R$. elongata, $R$. filiformis and L. griseus may differentially evoke apparent competition among the prey. In the aquatic community where these predators and prey coexist, mosquito larvae may benefit from apparent competition that reduces their vulnerability to predators. The identity of the alternative prey appears to be an important factor for shielding the vulnerability of mosquito prey to the generalist insect predators.
\end{abstract}

Key words: Heteroptera, mosquito, prey selectivity, apparent competition

\section{Introduction}

Alternative prey influences the outcome of prey predator interactions in aquatic communities. The presence of more than one prey creates an opportunity for the predator to exhibit selectivity, resulting in differential prey resource exploitation. Consequently, prey species can either benefit or suffer, relative to one another, as a part of the apparent competition (Holt 1977, 2012). Many theoretical (Holt and Kotler 1987; Strauss 1991; Holt and Lawton 1994; Wootton 1994) and empirical studies (Bonsall and Hassell 1997; Chaneton and Bonsall 2000; Morris et al. 2001, 2004) provide evidence in support of the coexistence of competing species through interactions with their shared pred- ators. Although apparent competition helps in the maintenance of prey diversity (Bonsall and Hassell 1997), the coexistence of multiple prey increases obstruction in target prey regulation (Morris et al. 2001, 2004; Liu et al. 2006). The invasion of exotic species (Settle and Wilson 1990) and the consequent displacement (Giovanelli et al. 2003) and reduction (Carvalheiro et al. 2008) of native species can also result from apparent competition.

The relevance of apparent competition extends to the context of biological control of vector mosquitoes by generalist predators (Blaustein and Chase 2007; Juliano 2009). Mosquito breeding sites such as rice fields and the associated wetlands harbour diverse insect predators, which consume a wide range of prey, including mosquito larva (Bam- 
baradeniya et al. 2004; Banerjee et al. 2010; Kundu et al. 2014). Consumption of alternative prey reduces the vulnerability of mosquito prey to the insect predators, affecting the regulation of vector mosquitoes. As a consequence of consumption by a shared predator, the competitive effect of alternative prey on the mosquito larvae is reduced, which may enhance the mosquito population similarly to the expected outcome of apparent competition. Although a wide dietary choice may favour generalist predators to sustain conditions with depleted prey, the efficacy to reduce target prey is affected. Empirical evidence indicates that alternative prey buffers the vulnerability of target mosquito prey regulation by guppy (Poecilia spp.) and mosquitofish (Gambusia spp.) (Bence 1988; Blaustein 1992; Manna et al. 2008, 2011; Kumar et al. 2015). Chironomid larvae, zooplankton and other alternative prey reduce mosquito prey consumption by insect predators like water bugs (Aditya et al. 2004; Saha et al. 2009, 2010; Sivagnaname 2009), odonate larvae (Saha et al. 2010, 2012) and dytiscid beetles (Aditya and Saha 2006) inhabiting tropical ponds and wetlands (Sunish and Reuben 2002; Bambaradeniya et al. 2004; Das et al. 2006). Even mosquito egg-raft predation varies in the presence of alternative prey and predator identity (Mukherjee and Blaustein 2019). In the majority of these studies, the insect predators exhibit mosquito prey selectivity against a single alternative prey, although it is more obvious that natural habitats will host multiple species as alternative prey. Since the extent of apparent competition may vary with the number and identity of alternative prey taxa, it is useful to compare the ability of a particular alternative prey when present separately or in groups. This would help to elucidate the efficiency of that particular alternative prey in reducing the predation pressure on the mosquito.

In order to justify these propositions, in the present study, mosquito prey selectivity of three predatory water bugs (Heteroptera: Nepidae), in the presence of single and multiple alternative prey were assessed under laboratory conditions. Insects belonging to the family Nepidae commonly occur in the wetland ecosystem together with mosquito larvae (Bambaradeniya et al. 2004; Das et al. 2006; Kundu et al. 2014). The general biology of these insects indicates their availability throughout the year. Apart from mosquito larvae, chironomid lar- vae, snails, fish fry and tadpoles are the common prey of these predatory insects (Ohba and Nakasuji 2006; Sano et al. 2011; Kloskowski et al. 2020). The predation ecology of the insects may vary in a context dependent manner (Juliano 2009), depending on the presence of the alternative prey (Saha et al. 2009, 2010, 2012), the habitat conditions (Saha et al. 2008, 2009; Dalal et al. 2019) and the search area (Dalal et al. 2020). A precise assessment of the functional role of the insects as predators of mosquitoes can be deduced through the study on prey consumption in the presence of alternative prey with the possibilities of several indirect interactions (Blaustein and Chase 2007), like apparent competition or intraguild predation (Barry and Roberts 2014; Kundu et al. 2014; Brahma et al. 2015a,b). In a similar way, the species-specific variations of the alternative prey in mosquito prey selection by the predatory insects will be an important aspect to judge the success in the biological control of mosquitoes.

Although the application of generalist insect predators for mosquito control has inherent limitations, these predators nevertheless enable natural regulation, preventing the ill effects of pesticides. The functional aspects of the predators reflect both the opportunity and the limitations of their use in the biological control programme (Dambach 2020). Considering conservation biological control as an opportunity, and the generalist predatory nature as a limitation for mosquito regulation, some insights about indirect interactions (Barry and Roberts 2014; Kundu et al. 2014; Brahma et al. 2015a,b) can be deduced through observation of the predation of nepid bugs in the present study. The selection of mosquito against one or multiple alternative prey by the insect predators will reflect the efficacy of insect predators in biological control of mosquitoes (Fig. 1). Similarly, the species-specific roles of each prey in reducing the vulnerability of the mosquito prey, separately, as well as in groups, will reflect the possibility of apparent competition. Thus, mosquito prey selectivity by nepid bugs and the role of the alternative prey remained the test hypothesis in the present study. 
A

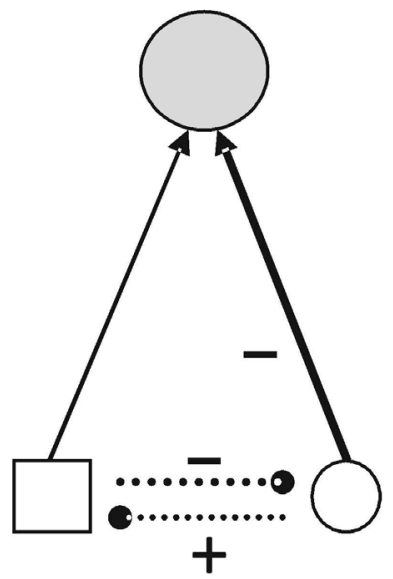

B

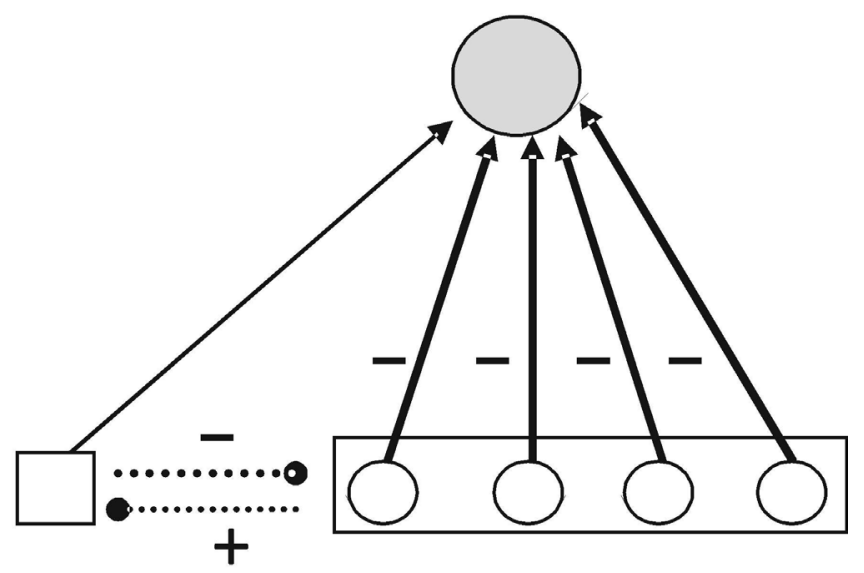

Fig. 1. Apparent competition and mosquito control using insect predators: the interactions between mosquito predator (filled circle) and target (mosquito) prey (square) in the presence of single (A) and multiple (B) alternative prey (open circles) and direct (straight lines) and indirect interactions (dotted lines) are shown. The strength of apparent competition of a particular alternative prey was expressed as a ratio in the single and multiple alternative prey system. The width weight of the arrows represents preference for the prey. Ability of alternative prey to lower the efficacy of mosquito control by the predators is expressed as the coefficient of apparent competition, and the individual strength of the alternative prey was evaluated in the present study

\section{Materials and methods}

\section{Predators: Collection and maintenance}

The insect predators (Heteroptera: Nepidae): Ranatra elongata Fabricius, 1790, Ranatra filiformis Fabricius, 1790, and Laccotrephes griseus (GuérinMéneville, 1835) were considered for the present study. They were collected from the East Calcutta Wetlands, Kolkata, India with the help of an insect net $(200 \mu \mathrm{m}$ mesh size). The samples collected comprised of a multi-species assemblage from which $R$. filiformis, $R$. elongata and L. griseus was initially segregated following order (Hemiptera), family (Nepidae) and genus characteristics (Thirumalai 1999). In the laboratory, the genus Ranatra was distinguished from Laccotrephes by its elongated, slender body, long, retractile appendages and terminal siphon. In contrast, Laccotrephes had an oblong shaped body, much shorter appendages and an acute terminal spine. The two species of Ranatra were differentiated on the basis of body length, $R$. elongata (ranged between 37 and $46 \mathrm{~mm}$ in body length) being much longer than $R$. filiformis (ranged between 22 and $28 \mathrm{~mm}$ in body length). The predators were maintained in the laboratory within glass aquaria containing 30 of sieved pond water with a few snails, fish fry, mosquito and chironomid larvae as food and aquatic plants such as Vallisneria spiralis L., Chara vulgaris L. as resting sites. The predators were collected at different intervals during the course of the experiments so that the population maintained in the laboratory comprised of randomly collected individuals. Once an individual predator had been used in a trial, it was placed within a separate plastic bucket to avoid its repetition in subsequent trials. No specific permissions were required for any locations / activities for the study. The study did not involve endangered or protected species. However, it was approved and complied with the norms of the Institutional Animal Ethics Committee of the Department of Zoology, University of Calcutta, Kolkata 700019, India. The use of the experimental animals, including collection and maintenance, complied with the norms of the Institutional Animal Ethical Committee, Department of Zoology, University of Calcutta, Kolkata, India.

\section{Prey: collection and maintenance}

The mosquito larvae Culex quinquefasciatus Say, 1823 (Diptera: Culicidae) were collected from sewage drains of the Ballygunge Science College Campus, Kolkata, India. Based on size, the collected larvae were separated as small ( $\sim$ II instar $1.4-2.3 \mathrm{~mm}$ in length, $0.9-1.3 \mathrm{mg}$ wet weight) and large ( IV instar $4.8-5.9 \mathrm{~mm}$ in length, $1.8-2.3 \mathrm{mg}$ 
wet weight) larvae. The smaller larvae were further reared in enamel trays containing de-chlorinated tap water and yeast powder as food (Laviest ${ }^{\circledR}$ capsules). The collections of larvae were made regularly to ensure continuous availability for the experiments.

The chironomid larvae were obtained from the sediment collected from the same sewage drains. The collected sediment was placed within enamel tubs containing sewage and tap water in equal volume, and the set-up was left undisturbed for 2-3 $\mathrm{h}$ under a light source. This allowed the chironomid larvae of varied species (Nandy et al. 2012) to emerge and cling to the sides of the tub, which were then collected with the help of a pipette and the larger ones $(>15 \mathrm{~mm})$ were used in the experiments.

The fry stages of the fish Labeo rohita Hamilton, 1822 (Cypriniformes: Cyprinidae) that were used in the experiments measured $3.2-3.5 \mathrm{~cm}$ in length from mouth to base of the caudal fin, and weighed $0.18-0.21 \mathrm{~g}$ (wet). These were bought from a local fish farm and maintained in the laboratory within plastic tubs (52 cm diameter and $24 \mathrm{~cm}$ in height) containing $70 \mathrm{dm}^{3}$ sufficiently aerated water, at low densities (100 individuals per $70 \mathrm{dm}^{3}$ ). The fry stages of the fish Labeo rohita were obtained from a commercial fish farm (M/S, Nilu Ghosh fish farm, Naihati, West Bengal, India) and following completion of the experiments, they were released into the ponds of the Ballygunge Science College campus, Ballygunge, Kolkata, India.

The tadpoles of Duttaphrynus melanostictus (Schneider, 1799) (Anura: Bufonidae) (formerly Bufo melanostictus) and the snails Racesina luteola (Lamarck, 1822) (Gastropoda: Lymnaeidae) (formerly Lymnaea luteola) were collected from the same location as that of the predators and maintained in the laboratory within the glass aquaria. Sediments, detritus and lettuce were added as food source. The 0-leg stage tadpoles weighing 10.7-12.4 mg and snails measuring 3-5 mm in shell height were considered as prey.

The predators and the prey were maintained within the laboratory under optimal conditions of temperature ranging between 25 and $30^{\circ} \mathrm{C}$, relative humidity between 80 and $85 \%$, and photoperiod $14 \mathrm{~h} \mathrm{~L}: 10 \mathrm{~h} \mathrm{D}$. The predators, before use in the experiments, were fed to satiation and starved for $24 \mathrm{~h}$ and only those predators that had been accli- matized to laboratory conditions for at least 7 days were used in the experiments. As food, the predators were provided with adequate numbers of all the prey types used in the experiment. The experiments were carried out between June and September of 2012 during the rainy season, with an abundance of all the predator and prey species in the freshwater habitats. Following completion of the experiments, all the insect predators and tadpoles were released into the ponds of the Ballygunge Science College campus, Ballygunge, Kolkata, India.

\section{Experimental design}

Two-prey system: Mosquito larvae were paired with one type of alternative prey (chironomid larvae, fish fry, snails or tadpoles) in a 30:15 ratio (except mosquito: chironomid, where the ratio was 30:30) and offered to a single predatory insect.

Multi-prey system: A single predator was exposed to mosquito, chironomid, fish fry, tadpoles and snails together in the same medium in a ratio of 30:30:15:15:10. Six trials were repeated per predator species per prey combination for both systems (Klecka and Boukal 2012). The experimental system included a glass aquarium $(38 \times 36 \times 36 \mathrm{~cm})$ filled with $35 \mathrm{dm}^{3}$ of water (aged tap water: pond water $=1: 1 \mathrm{pH} 7.5-8.0$ depending on the pond water used during the course of the study) with several specimens of Pistia, Ipomoea and Lemna as refuge for both prey and predators. Table 1 illustrates the outline of the experimental design. The number of each type of prey consumed was noted after $24 \mathrm{~h}$ and the data was used to calculate preference using the formula (Chesson 1983): $\alpha_{\mathrm{i}}=\left[\ln \left(n_{i 0}-r_{i}\right) / n_{i 0}\right]$ / $\left[\sum \ln \left(n_{j 0}-r_{j}\right) / n_{j 0}\right]$, where, $\alpha_{i}=$ Manly's selectivity index for prey type $i, n_{i 0}=$ number of items of type $\mathbf{i}$ present at the start of the experiment; $r_{i}=$ number of prey type $i$ consumed; $r_{j}=$ number of total prey type $j$ consumed, $n_{j 0}=$ number of total items of type $j$ present at the start of the experiment, $j=$ 1 to $m$ types of prey, $m=$ number of prey types = 5 (fish fry, snail, tadpole, chironomid and mosquito larvae). The value of $\alpha_{i}$ ranges from 0 to 1 . In the 2-prey system $\alpha_{i}=0.5$ indicates non-selective feeding, $\alpha_{i}>0.5$ indicates positive selection, $\alpha_{i}<0.5$ indicates negative selection. Similar values for $\alpha_{i}$ in the presence of five prey items would be 0.2 . Error values of alpha were then obtained from the mean and bootstrapped confidence interval $(\mathrm{CI})$. The de- 
viations of alpha from non-selective feeding were obtained by calculating bootstrapped $\mathrm{Cl}$ (Efron and Tibshirani 1991) using $R$ software (boot, boot.ci, "basic" bootstrapping for confidence intervals, $R=$ 9,999) (Davison and Hinkley 1997; Canty and Ripley 2020) to determine if the confidence interval overlaps non selective feeding $(0.5$, for 2 prey; 0.2 , for multiprey) or each other.

The diet overlaps of the three predatory insects were calculated using Pianka's overlap index in Ecosim 7.0 (Gotelli and Entsminger 2001) based on the formula: $\mathrm{O}_{\mathrm{ij}}=\mathrm{O}_{\mathrm{ji}}=\sum \alpha_{\mathrm{ik}} \alpha_{\mathrm{jk}} / \sqrt{ } \alpha_{\mathrm{ik}}^{2}{ }_{\mathrm{jk}}^{2}$, where $\mathrm{O}_{\mathrm{ij}}=$ $\mathrm{O}_{\mathrm{ji}}$ denotes diet overlaps of predator species $\mathrm{j}$ and $\mathrm{i}$, the values denote Manly's selectivity for a particular prey. The value of $\mathrm{O}_{\mathrm{ij}}=\mathrm{O}_{\mathrm{ji}}$ lies between 0 and 1 , the greater the value, the more similar the diet between the predator pairs.

The ratio of selectivity for mosquito in the two-prey $\left(\alpha_{M 2}\right)$ and multi-prey $\left(\alpha_{M 5}\right.$, M5 since five prey were present) system was used to represent the coefficient of apparent competition (CAC), $k$, using the formula: $\alpha_{\mathrm{M} 2}=k \times \alpha_{\mathrm{M} 5}$.

The value of $k$ reflects the impact of an alternative prey against mosquito when present in twoprey and multiple prey conditions, and thus $k$ was used as a surrogate of apparent competition attributable to the alternative prey. A value of $k=1$, indicates no difference in impact by the alternative prey in the two prey against multiple prey condition; for a particular alternative prey, the smaller the value of $k$ from 1 , the greater will be its ability to depress the preference for mosquito. The one tailed $t$-test (Zar 1999 ) was performed to confirm if the values of $k$ differed significantly from 1 . The statistical analyses were conducted using SPSS 17.

The experimental trials were carried out at

Table 1 . Outline of the experimental design followed in the present study

Experiment Remarks

\section{A. Two prey experiment}

\begin{tabular}{|c|c|c|c|c|}
\hline Prey used & $\begin{array}{l}\text { Chironomid larvae } \\
\text { (C) }\end{array}$ & $\begin{array}{l}\text { Aquatic snails } \\
\text { (AS) }\end{array}$ & $\begin{array}{l}\text { Fish fry } \\
\text { (FF) }\end{array}$ & Tadpoles $(\mathrm{T})$ \\
\hline Predators & \multicolumn{4}{|c|}{ Laccotrephes griseus, Ranatra elongata, Ranatra filiformis } \\
\hline Prey ratio [Mosquito(M) : Alternative prey] & $1: 1$ & $2: 1$ & $2: 1$ & $2: 1$ \\
\hline Prey density & 60 & 45 & 45 & 45 \\
\hline Trials / Predators & \multicolumn{4}{|c|}{$6 \times$ prey ratio $\times 4$ alternative prey $=72$ trials } \\
\hline
\end{tabular}

\section{B. Multiple prey experiment}

\begin{tabular}{cccccc}
\hline Prey used & M & C & AS & FF & T \\
Prey combination & 6 & 6 & 3 & 3 & 2 \\
Prey density & 30 & 30 & 15 & 15 & 10 \\
\hline Trials / Predators & \multicolumn{5}{c}{6 trials $\times 1$ prey combination } \\
& (total $=18$ trials for the 3 preclator species) \\
\hline
\end{tabular}

Predators

Observation

Analysis

Hypothesis tested
Laccotrephes griseus, Ranatra elongata, Ranatra filiformis

Number of live prey present was observed and counted at the end of $24 \mathrm{~h}$ period.

Manly's alpha selectivity index and comparison between the values in the two-prey and multiple-prey experiment along with the Pianka's niche overlap index.

Relative efficacy of the alternative prey to reduce the vulnerability of the mosquito larvae when present as a sole alternative prey and with multiple alternative prey. 
different time intervals using different prey and predator individuals, such that the trials represent true replicates. Irrespective of species, the prey and predator individuals once used in the experiments were discarded without further maintenance in the laboratory. For a particular trial, the selection of prey and predator individuals was made at random from the pool of individuals maintained in the laboratory, to randomize the selection of prey and predator individuals. For a particular experiment, the trials were conducted at definite time intervals, in accordance with "interspersion" (Hurlbert 1984).

\section{Results}

Mosquito prey consumption by the insect predators varied with the two prey and multiple prey system (Table 2 ). In all instances the selectivity for the mosquito larvae was influenced by the presence of alternative prey. In the presence of snails and tadpoles as single alternative prey, $R$. elongata, $R$. filiformis and L. griseus exhibited a strong positive selectivity for mosquito larvae (Fig. 2). In other words, snails and tadpoles were least preferred by all the three predatory insects. The preference pattern for mosquito was different in the presence of chironomid and fish fry as alternative prey. In the presence of chironomid larvae, $R$. elongata and $L$. griseus preferred mosquito larvae $\left(\alpha_{\mathrm{m}}>0.5\right)$, but $R$. filiformis exhibited an opportunistic pattern $\left(\alpha_{\mathrm{m}}\right.$ $\sim 0.5)$. In the presence of fish fry, $R$. filiformis exhibited negative selectivity for mosquito larvae, the pattern being opposite for the two other predators. In the multi-prey system, $R$. elongata and $L$. griseus positively selected mosquito larvae. These two predators also showed preference for fish fry but none seemed to prefer chironomid larvae, the avoidance being stronger for L. griseus. On the other hand, $R$. filiformis exhibited a distinct preference for fish fry over all the other prey types including mosquito larvae, a pattern which was also evident in the two prey treatments. Apparently, all the insect predators tended to avoid snails and tadpoles, both under two prey and multiple prey conditions (Figs. 2 and 3). The observed mean overlap index $\left(\mathrm{O}_{\mathrm{ij}}\right)$ value was 0.77 and differed significantly $(p$ $<0.02$ ) from the expected value of 0.46 (mean of simulated indices). The diet overlaps for predator pairs, as observed from Pianka's index varied from 0.53 (R. elongata and R. filiformis) to 0.93 (R. filifor- mis and L. griseus) (Table 2).

The $k$ values (Table 3 ) quantified the effect of a particular alternative prey on the preference for the target prey (mosquito larvae). The $k$ values indicated that in the case of $R$. elongata, chironomid larvae had the greatest impact on $\alpha_{M}$ (preference for mosquito), followed by fish fry, tadpoles and snails, although the deviation of the $k$ value from 1 was not significant $(p>0.05)$ for chironomid larva. In the case of $R$. filiformis and L. griseus the interaction with mosquito larvae was maximally impacted by fish fry followed by chironomids, tadpoles and snails. This is further elaborated in Fig. 4, where the relative contribution of the alternative prey is shown with reference to the three nepid water bugs.

\section{Discussion}

Different species of water bugs consume mosquito larvae in varying numbers depending on their taxonomic identity and habitat conditions (Aditya et al. 2004; Saha et al. 2009, 2010; Sivagnaname 2009). However, the prey consumption pattern of $R$. elongata, R. filiformis and L. griseus was lower in comparison to observations of the other co-occurring water bugs (Belostomatidae) namely, Diplonychus annulatus (Fabricius), D. rusticus (Fabricius) and Anisops bouvieri (Kirkaldy) (Aditya et al. 2004; Saha et al. 2007a, b, 2009, 2010). In the water bugs Kirkaldyia (= Lethocerus) deyrollei (Vuillefroy) (Belostomatidae) and Appasus japonicus Vuillefroy (Belostomatidae) the differences in the shape of the rostrum and the claws (Ohba 2008) have been attributed to their differences in prey selection pattern and dietary choice (Ohba and Nakasuji 2006). In many heteropteran bugs exploitation of the space and prey consumption vary with the availability of light (Saha et al. 2008), habitat complexity (Saha et al. 2009), and relative abundance and orientation of prey (Aditya et al. 2005; Sivagnaname 2009, Saha et al. 2010) in the habitat. Similarly, among the nepid water bugs considered in the present study, the variations in prey consumption were likely due to differences in morphological features such as rostrum and legs. The prey consumption pattern and the predatory tactics of nepid bugs are similar to those of dragonfly larvae, following a sit-and-wait strategy (Flynn and Moon 2011), in comparison to the belostomatid bugs and diving beetles, which actively hunt for prey (Saha et al. 2007a,b; Ohba 
Table 2. Prey consumption (Mean \pm SE) by the three different heteropteran predators $R$. elongata, $R$. filiformis and L. griseus under in two prey (A) and multiple prey (B) conditions along with the Pianka's niche overlap index (C) based on the consumption of different prey species

A. Two prey experiment

\begin{tabular}{|c|c|c|c|c|}
\hline \multirow{2}{*}{\multicolumn{2}{|c|}{ Prey species }} & \multicolumn{3}{|c|}{ Predator } \\
\hline & & Ranatra elongata & Ranatra filiformis & Laccotrephes griseus \\
\hline \multirow{8}{*}{ Prey combination } & Mosquito larva & $20.17 \pm 2.94$ & $9.50 \pm 0.76$ & $19.17 \pm 2.94$ \\
\hline & Chironomid larva & $16.00 \pm 1.77$ & $8.50 \pm 0.42$ & $4.00 \pm 0.68$ \\
\hline & Mosquito larva & $21.16 \pm 2.61$ & $8.67 \pm 0.92$ & $14.83 \pm 0.91$ \\
\hline & Fish fry & $3.33 \pm 0.49$ & $6.67 \pm 0.42$ & $4.67 \pm 0.33$ \\
\hline & Mosquito larva & $25.5 \pm 0.76$ & $13.16 \pm 2.72$ & $18.17 \pm 2.46$ \\
\hline & Aquatic snail & $0.83 \pm 0.31$ & $0.17 \pm 0.17$ & $0.33 \pm 0.21$ \\
\hline & Mosquito larva & $13.67 \pm 1.86$ & $15.67 \pm 1.49$ & $13.17 \pm 2.30$ \\
\hline & Tadpole & $0.33 \pm 0.21$ & $0.17 \pm 0.17$ & $0.17 \pm 0.17$ \\
\hline
\end{tabular}

B. Multiple prey experiment

\begin{tabular}{cccccc}
\hline \multirow{2}{*}{$\begin{array}{c}\text { Predator } \\
\text { Mosquito larva }\end{array}$} & Chironomid larva & Fish fry & Aquatic snail & Tadpole \\
Ranatra elongata & $19.50 \pm 2.29$ & $9.50 \pm 1.23$ & $5.67 \pm 1.14$ & $1.17 \pm 0.31$ & $0.67 \pm 0.33$ \\
Ranatra filiformis & $9.83 \pm 2.73$ & $4.00 \pm 1.31$ & $13.00 \pm 0.36$ & $2.17 \pm 0.47$ & $0.33 \pm 0.21$ \\
Laccotrephes griseus & $12.83 \pm 1.05$ & $2.83 \pm 0.47$ & $8.83 \pm 1.19$ & $1.17 \pm 0.31$ & $0.33 \pm 0.21$ \\
\hline \multicolumn{7}{c}{ C. Values of Pianka's niche overlap index } \\
Ranatra elongata & Ranatra filiformis & Laccotrephes griseus \\
Ranatra filiformis & 0.56 & 0.81 \\
\hline
\end{tabular}

and Takagi 2010). The sit-and-wait strategy is characteristic of Ranatra (Blois and Cloarec 1983; Bailey 1986) that require that the prey be pursued once it arrives in the vicinity of the predator. Under natural conditions nepid predators exhibit slow mobility compared to the belostomatid bugs and diving beetles, more suitable for their sit-and-wait strategy.

From the results of the two-prey and multiple-prey experiments, it was apparent that the presence of alternative prey influenced the mosquito prey selectivity of the predatory insects. Selectivity of alternative prey by $R$. filiformis, $R$. elongata and $L$. griseus varied with prey species identity. As indicated through the coefficient of apparent competition, $k$, chironomid larvae, tadpoles, snails and fish fry as alternative prey, buffered the vulnerability of target mosquito prey with varying intensity. For instance, L. griseus and R. filiformis showed higher selectivity for fish fry while $R$. elongata preferred chironomid larvae. Effectively, the differences in the prey selection pattern are a reflection of resource partitioning among the three species $R$. elongata, $R$. filiformis and L. griseus that co-occur in the wetlands. The alternative prey species were observed to exert a varying competitive effect depending on the predator species, as indicated by the values of coefficient of apparent competition (Table 3). Thus, chironomids had a higher impact on mosquito prey selectivity of $R$. elongata than of $R$. filiformis and $L$. griseus. A similar effect was exerted by fish fry on the prey selectivity of $R$. filiformis and $L$. griseus. The preference for alternative prey displayed by predatory insects raises a concern for the effective regulation of the mosquito population, since it lowers the possibility of selection of mosquito prey in the diet (Blaustein and Chase 2007; Juliano 2009). Although alternative prey consumption is common for generalist predatory insects like odonate larvae (Saha et al. 2009), diving beetles (Aditya and Saha 2006), 
Table 3. Values of " $k$ " (coefficient of apparent competition) calculated from the equation $\alpha_{\text {M2-prey }}=k \times \alpha_{\text {Mmulti-prey }}$, where $k$ is a measure of the effect of a particular alternative prey on the selectivity of mosquito under two-prey in contrast to multiple prey conditions. In this instance, a value of $k=1$, would indicate no difference in mosquito prey selectivity under two prey or multiple prey conditions due to the particular prey. The smaller the value of $k$ for a particular alternative prey, the greater will be its ability to depress the preference for mosquito. Values of $k$ significantly different from 1 are marked with asterisk (results of $t$-test, $\mathrm{p}<0.05$ ). Here, chironomid larvae have the highest impact on $\alpha_{M}$ for the predator Ranatra elongata whereas fish fingerlings depress $\alpha_{M}$ the most in the case of Ranatra filiformis and Laccotrephes griseus

\begin{tabular}{cccc}
\hline \multirow{2}{*}{ Alternative prey } & \multicolumn{3}{c}{ Predator } \\
\cline { 2 - 4 } & Ranatra elongata & Ranatra filiformis & Laccotrephes griseus \\
\hline Chironomid larva & 1.1 & $3.6^{*}$ & $2.0^{*}$ \\
Fish fry & $1.5^{*}$ & $2.4^{*}$ & $1^{*}$ \\
Snail & $1.8^{*}$ & $6.5^{*}$ & $2.8^{*}$ \\
Tadpole & $1.7^{*}$ & $6.5^{*}$ & $2.8^{*}$ \\
\hline
\end{tabular}

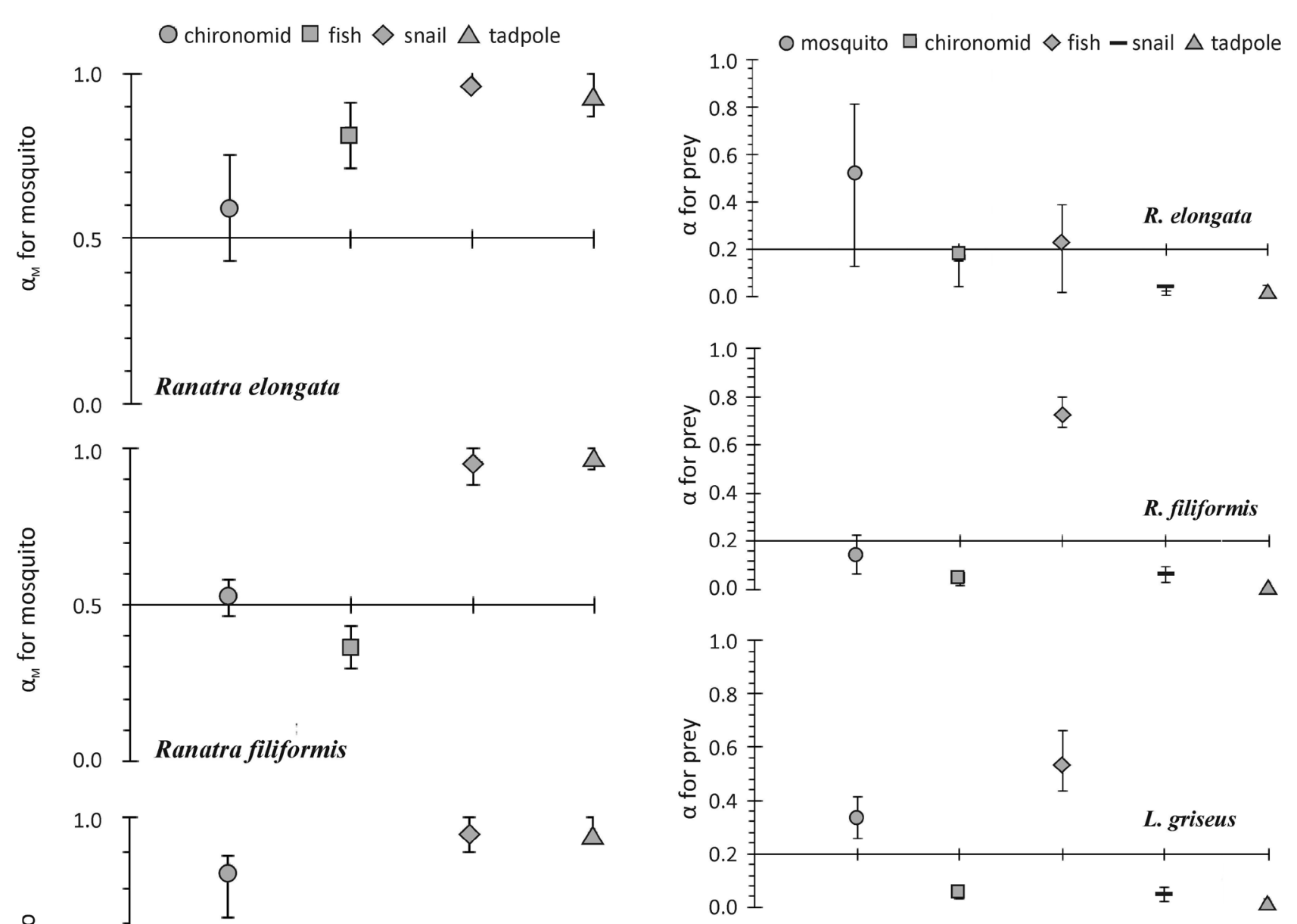

Fig. 3. Manly's selectivity index ( $\alpha$-alpha) for mosquito larvae, chironomid larvae, fish fry, snails and tadpoles in multi-prey system for $R$. elongata, $R$. filiformis and L. griseus. Selectivity of 0.2 indicates no preference or consumption at rates proportional to the abundance of each prey in the medium. Confidence intervals were obtained through bootstrapping

$0.0 \perp$ Laccotrephes griseus

Fig. 2. Manly's selectivity index $(\alpha M)$ for mosquito larvae in the presence of chironomid larvae, fish fingerlings, snails and tadpoles as alternative prey in separate treatments. Selectivity of 0.5 indicates no preference or consumption at rates proportional to each prey's abundance in the medium. Symbols show averages (six replicates per each prey combination) and whiskers represent confidence intervals obtained through bootstrapping 

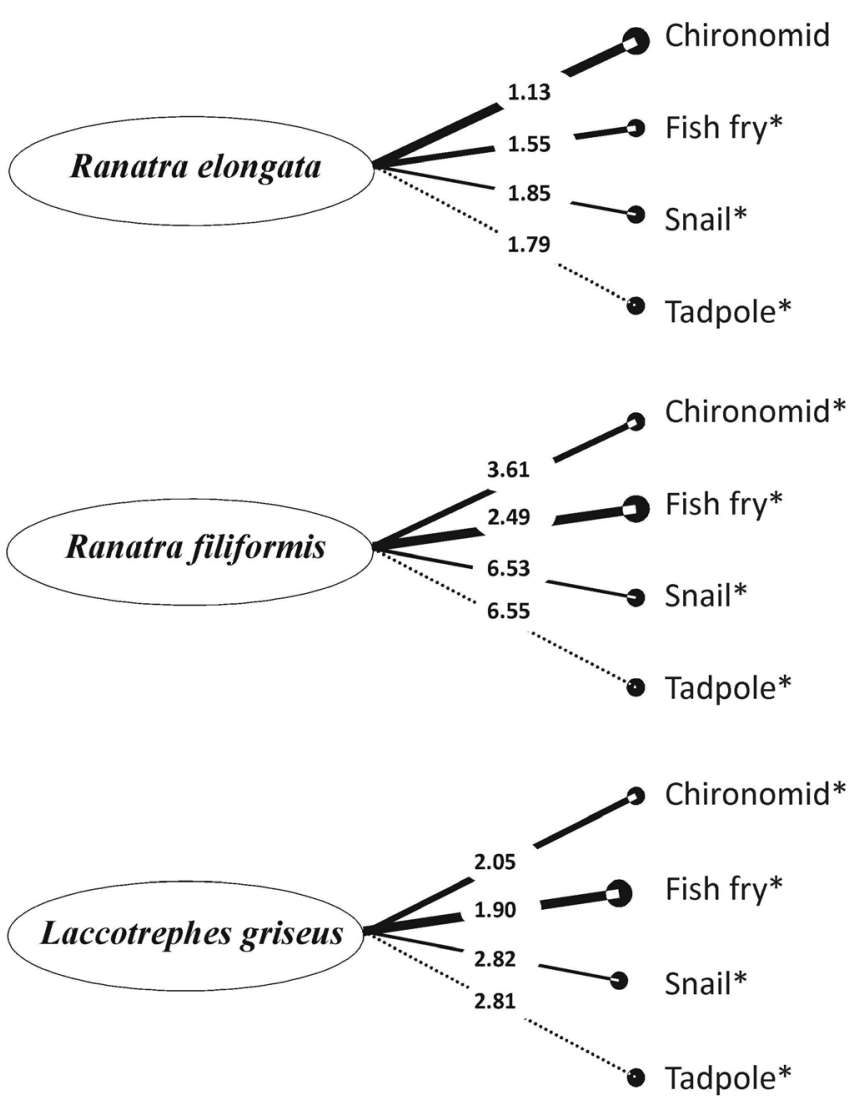

Fig. 4. Schematic representation of the links of the prey to the three water bugs (Nepidae), justifying the generalist predatory nature. The thickness of the arrows indicates the magnitude of impact each alternate prey exerts on the selectivity of mosquito. Here, chironomid larvae have the highest impact on $\alpha M$ for the predator $R$. elongata whereas, fish fingerlings depress $\alpha M$ the most in the case of $R$. filiformis and L. griseus, as mentioned in Table 3

and heteropteran bugs (Saha et al. 2008, 2010), the relative efficiency of the alternative prey to reduce mosquito prey mortality varies with the prey preference of the concerned species. Proximately, based on the relative number of alternative prey available, the efficacy of a particular predator may vary accordingly in different mosquito larval habitats. The spatial orientation and mobility of the prey influence their vulnerability to the insect predators, as a result of which the prey selectivity may vary. The movement and distribution of chironomid larvae, snails, fish fry and tadpoles differ from mosquito larvae, which may have influenced the prey selection by the insect predators. Although, at a proximate level, the efficacy of the nepid water bugs may vary with the relative number of the alternative prey available, the microhabitat utilization and spatial orientation of the prey are also crucial factors in the outcome of the species interaction.

Species composition in mosquito breeding sites varies according to size, location and habitat permanence (Sunahara et al. 2002; Banerjee et al. 2010). Considering tropical wetlands as mosquito breeding sites, multiple prey species are available for the insect predators $R$. elongata, $R$. filiformis and L. griseus (Bambaradeniya et al. 2004). Theory suggests that alternative prey can induce apparent mutualism, reducing predation pressure on mosquito larvae (Juliano 2009). Apparent mutualism is a form of apparent competition, viewed as a benefit to the prey population when they occur together and share a common predator (Blaustain and Chase 2007). Mosquitoes will benefit from the selection of one or multiple alternative prey by these insect predators, resulting in unaltered mosquito population growth even in presence of the insect predators. Although long-term assessment is essential to judge the population consequences of prey selectivity by nepid bugs and other insect predators (Bailey 1986; Ohba and Nakasuji 2006; Ohba 2008; Klecka and Boukal 2012), the present instance utilized the prey selectivity index as a surrogate to highlight the effects of apparent competition on the mosquito population. The prey selectivity pattern of the nepid water bugs suggests apparent completion as an obvious mechanism to reduce mosquito prey vulnerability (Kundu et al 2014). Given that the preservation of the ecosystem services of wetlands is a priority, generalist insect predators such as $R$. elongata, R. filiformis and L. griseus deserve promotion for regulation of mosquitoes using natural enemies. However, success with the use of the nepid bugs as a biocontrol agent may be constrained through indirect interactions like apparent competition and intraguild predation (Brahma et al. 2015a,b). The selection and relative preference for alternative prey constitute an inherent limitation with the use of generalist insect predators, including these nepid bugs, in biological control of mosquitoes (Saha et al. 2009, 2010, 2012). On the other hand, the use of insect predators for biological control of mosquitoes would augment the conservation biological control mechanism of preserving ecosystem functions and regulating mosquitoes using natural enemies. Thus, predatory insects such as these nepid bugs can impose constraints and limitations I (Dambach 2020) in achieving the 
goal of mosquito regulation effectively. Further field tests using these predators and the constraints that prevent the regulation of mosquitoes (Kundu et al. 2014; Brahma et al. 2015a,b) need to be evaluated to substantiate the proposition of using generalist insect predators for regulation of mosquitoes on wetlands.

\section{Acknowledgements}

The authors acknowledge the constructive comments of the esteemed reviewers in enhancing the manuscript to the present form. The authors are grateful to the respective Heads, Department of Zoology, University of Calcutta and University of Burdwan, India, for the facilities provided including the DST-FIST and UGC-DRS SAP programmes, Government of India. GA acknowledges UGC for the Research Award sanction no. F.30-90(SA-II) /2009, dated 17 Sep. 2009.

\section{Authors' contributions}

Conceived and designed: G.A., G.K.S.; Field observations and experimental execution: N.S., M.K., G.A.; Statistical analysis: N.S., G.A.; Compilation: G.A., M.K., N.S., G.K.S.

\section{References}

Aditya G., Bhattacharyya S., Kundu N., Saha G.K., 2005, Frequency-dependent prey-selection of predacious water bugs on Armigeres subalbatus immatures, J. Vector Borne Dis. 42(1): 9-14.

Aditya G., Bhattacharyya S., Kundu N., Saha G.K., Raut S.K., 2004, Predatory efficiency of the water bug Sphaerodema annulatum on mosquito larvae Culex quinquefasciatus and its effect on adult emergence, Biores. Technol. 95(2): 169-172.

Aditya G., Saha G.K., 2006, Predation of the beetle Rhantus sikkimensis (Coleoptera: Dytiscidae) on the larvae of Chironomus Meigen (Diptera: Chironomidae) of the Darjeeling Himalayas of India, Limnologica 36(4): 251-257.

Bailey P.C.E., 1986, The feeding behaviour of a sit-andwait predator, Ranatra dispar, (Heteroptera: Nepidae): description of behavioural components of prey capture, and the effect of food deprivation on predator arousal and capture dynamics, Behaviour 97(1): 66-93.

Bambaradeniya C.N.B., Edirisinghe J.P., De Silva D.N., Gunatilleke C.V.S., Ranawana K.B., Wijekoon S., 2004, Biodiversity associated with an irrigated rice agro-ecosystem in Sri Lanka, Biodiv. Conserv. 13(9): 1715-1753.

Banerjee S., Aditya G., Saha N., Saha G.K., 2010, An assessment of macroinvertebrate assemblages in mosquito larval habitats - space and diversity relationship, Environ. Monit. Assess.168(1-4): 597-611.

Barry M.J., Roberts D.M., 2014, Indirect interactions limit the efficiency of Odonata as natural control agents for mosquito larvae, J. Insect Behav. 27(5): 626-638.

Bence J.R., 1988, Indirect effects biological control of mosquitoes by mosquito fish, J. Appl. Ecol. 25(2): 505-521.

Blaustein L., 1992, Larvivorous fishes fail to control mosquitoes in experimental plots, Hydrobiologia 232(3): 219-232.

Blaustein L., Chase J.M., 2007, Interactions between mosquito larvae and species that share the same trophic level, Annu. Rev. Entomol. 52(1): 489-507.

Blois C., Cloarec A.,1983, Density-dependent prey selection in the water stick insect, Ranatra linearis (Heteroptera), J. Anim. Ecol. 52(3): 849-866.

Bonsall M.B., Hassell M.P., 1997, Apparent competition structures ecological assemblages, Nature 338: 371-373.

Brahma S., Sharma D., Kundu M., Saha N., Saha G.K., Aditya G., 2015a, Mosquito Prey vulnerability in intraguild predation between Ranatra filiformis and $A n$ isops bouvieri: implications in biological control, Proc. Zool. Soc. 68(1): 36-44.

Brahma S., Sharma D., Kundu M., Saha N., Saha G.K., Aditya G., 2015b, Intraguild predation in Heteroptera: effects of density and predator identity on dipteran prey, Neotrop. Entomol. 44(4): 374-384.

Canty A., Ripley B.D., 2020, Boot: Bootstrap R (S-Plus) functions. $R$ package version 1.3-25 [Computer software].

Carvalheiro L.G., Buckley Y.M., Ventim R., Fowler S.V., Memmott J., 2008, Apparent competition can compromise the safety of highly specific biocontrol agents, Ecol. Lett. 11(7): 690-700.

Chaneton E.J., Bonsall M.B., 2000, Enemy-mediated apparent competition: empirical pattern and the evidence, Oikos 88(2): 380-394.

Chesson J., 1983, The estimation and analysis of preference and its relationship to foraging models, Ecology 64(5): 1297-1304.

Dalal A., Cuthbert R.N., Dick J.T.A., Gupta S., 2019, Water depth-dependent notonectids predatory impacts across larval mosquito ontogeny, Pest Manag. Sci. 75(10): 2610-2617.

Dalal A., Cuthbert R.N., Dick J.T.A., Gupta S., 2020, Prey preferences of notonectids towards larval mosquitoes across prey ontogeny and search area, Pest Manag. Sci. 76(2): 609-616.

Dambach P., 2020, The use of aquatic predators for larval control of mosquito disease vectors: opportunities and limitations, Biol. Contr. 150: e104357. 
Das P.K., Sivagnaname N., Amalraj D.D., 2006, Population interactions between Culex vishnui mosquitoes and their natural enemies in Pondicherry India, J. Vector Ecol. 31(1): 84-88.

Davison A.C., Hinkley D.V.,1997, Bootstrap methods and their applications, Cambridge University Press, Cambridge, $582 \mathrm{pp}$.

Efron B., Tibshirani R.,1991, Statistical data analysis in the computer age, Science 253(5018): 390-395.

Flynn K.E., Moon D.C., 2011, Effects of habitat complexity, prey type, and abundance on intraguild predation between larval odonates, Hydrobiologia 675(1): 97-104.

Giovanelli A., Vieira M.V., da Silva C.L.P.A., 2003, Apparent competition through facilitation between $\mathrm{Me}$ lanoides tuberculata and Biomphalaria glabrata and the control of systematic, Mem. Inst. Oswaldo Cruz 98(3): 429-431.

Gotelli N.J., Entsminger G., 2001, EcoSim: Null models software for ecology. Version 7.0 [Computer software], Acquired Intelligence Inc. \& Kesey-Bear, Jericho, Vermont. Retrieved from: http:/homepages.together. net/ gentsmin/ecosim/ecosim. htm.

Holt R.D., 1977, Predation, apparent competition, and the structure of prey communities, Theor. Popul. Biol. 1292): 197-229.

Holt R.D., 2012, Apparent competition, [in:] Hastings A., Gross L.J. (eds), Encyclopedia of theoretical ecology, University of California Press, Berkeley: 45-52.

Holt R.D., Kotler B.P., 1987, Short term apparent competition, Am. Nat. 130(3): 412-430.

Holt R.D., Lawton J.H.,1994, The ecological consequences of shared natural enemies, Annu. Rev. Ecol. Syst. 25(1): 495-520.

Hurlbert S.H., 1984, Psedoreplication and the design of ecological field experiments, Ecol. Monogr. 54(2): 187-211.

Juliano S.A., 2009, Species interactions among larval mosquitoes: context dependence across habitat gradients, Annu. Rev. Entomol. 54(1): 37-56.

Klecka J., Boukal D.S., 2012, Who eats whom in a pool? A comparative study of prey selectivity by predatory aquatic insects, PLoS ONE 7(6): e37741.

Kloskowski J., Nieoczym M., Stryjecki R. 2020, Between-habitat distributions of pond tadpoles and their insect predators in response to fish presence, Hydrobiologia 847(5): 1343-1356.

Kumar R., Muhid P., Dahms H-U., Sharma J., Hwang J-S., 2015, Biological mosquito control is affected by alternative prey, Zool. Stud. 54: e55.

Kundu M., Sharma D., Brahma S., Pramanik S., Saha G.K., Aditya G., 2014, Insect predators of mosquitoes of rice fields: portrayal of indirect interactions with alternative prey, J. Entomol. Zool. Stud. 2(5): 97-103.

Liu C-Z., Yan L., Li H-R., Wang G., 2006, Effects of predator mediated apparent competition on the population dynamics of Tetranychus utricae on apples, BioControl
51(4): 453-463.

Manna B., Aditya G., Banerjee S., 2008, Vulnerability of the mosquito larvae to the guppies (Poecilia reticulata) in the presence of alternative preys, J. Vector Borne Dis. 45(3): 200-206.

Manna B., Aditya G., Banerjee S., 2011, Habitat heterogeneity and prey selection of Aplocheilus panchax: an indigenous larvivorous fish, J. Vector Borne Dis. 48(3): 144-149.

Morris R.J., Lewis O.T., Godfray H.C.J., 2004, Experimental evidence for apparent competition in a tropical forest food web, Nature 428(6980): 310-313.

Morris R.J., Muller C.B., Godfray H.C.J., 2001, Field experiments testing for apparent competition between primary parasitoids mediated by secondary parasitoids, J. Anim. Ecol. 70(2): 301-309.

Mukherjee S., Blaustein L., 2019, Effects of predator type and alternative prey on mosquito egg raft predation and destruction, Hydrobiologia 846(1): 215-221.

Nandy S., Aditya G., Saha G.K., 2012, Nutrient condition and chironomid assemblages in Kolkata, India: assessment for biomonitoring and ecological management, J. Limnol. 71(2): 320-329.

Ohba S.-Y., 2008, The number of tadpoles consumed by the nymphs of the giant water bug Kirkaldyia deyrolli under laboratory conditions, Limnology 9(1): 71-73.

Ohba S.-Y., Nakasuji F., 2006, Dietary items of predacious aquatic bugs (Nepoidea: Heteroptera) in Japanese wetlands, Limnology 7(1): 41-43.

Ohba S.-Y., Takagi M., 2010, Predatory ability of adult diving beetles on the Japanese encephalitis vector Culex tritaeniorhynchus, J. Am. Mosq. Contr. Assoc. 26(1): 32-36.

Saha N., Aditya G., Bal A., Saha G.K., 2007a, Comparative study of functional response of common Hemipteran bugs of East Calcutta Wetlands, India, Int. Rev. Hydrobiol. 92(3): 242-257.

Saha N., Aditya G., Bal A., Saha G.K. 2007b, A comparative study of predation of three aquatic heteropteran bugs on Culex quinquefasciatus larvae, Limnology 8(1): 73-80.

Saha N., Aditya G., Bal A., Saha G.K., 2008, Influence of light and habitat on predation of Culex quinquefasciatus (Diptera: Culicidae) larvae by the water bugs (Hemiptera: Heteroptera), Ins. Sci. 15(5): 461-469.

Saha N., Aditya G., Banerjee S., Saha G.K., 2012, Predation potential of odonates on mosquito larvae: Implications for biological control, Biol. Contr. 63(1): 1-8.

Saha N., Aditya G., Hampton S., Saha G.K., 2010, Opportunistic foraging by heteropteran mosquito predators, Aquat. Ecol. 44(1): 167-176.

Saha N., Aditya G., Saha G.K., 2009, Habitat complexity reduces vulnerability of preys: an experimental analysis using aquatic insect predators and immature dipteran prey, J. Asia Pacific Entomol.12(4): 233-239.

Sano K., Miyoshi K., Ishikawa S., Liepvisay N., Kurokura S., 2011, Impact of predation by water insects on fish 
seed production in Lao PDR, Jpn Agri. Res. Q. 45(4): 461-465.

Settle W.H., Wilson L.T., 1990, Invasion of variegated leaf hopper and biotic interactions: parasitism, competition and apparent competition, Ecology 71(4): 1461-1470.

Sivagnaname N., 2009, Selective and frequency dependent predation of aquatic mosquito predator Diplonychus indicus Venkatesan \& Rao (Hemiptera: Belostomatidae) on immature stages of three mosquito species, Entomol. Res. 39(6): 356-363.

Strauss S.Y., 1991, Indirect effects in community ecology - their definition, study and importance, Trends Ecol. Evol. 6(7): 206-210.

Sunahara T., Ishizaka K., Mogi M., 2002, Habitat size: a factor determining the opportunity for encounters between mosquito larvae and aquatic predators, J. Vector Ecol. 27(1): 8-20.

Sunish I.P., Reuben R., 2002, Factors influencing the abundance of Japanese encephalitis vectors in rice fields in India - II. Biotic, Med. Vet. Entomol. 16(1): 1-9.

Thirumalai G., 1999, Aquatic and semi-aquatic Heteroptera of India, Indian Association of Aquatic Biologists (IAAB) Publ. No. 7: 1-74.

Wootton J.T., 1994, The nature and consequences of indirect effects in ecological communities, Annu. Rev. Ecol. Syst. 25: 443-466.

Zar J.H., 1999, Biostatistical analysis, Prentice Hall, Upper Saddle River, 663 pp. 